\title{
MÉTODO PARA APLICAR LA TRILOGÍA DE VALORES MORALES DE SCHMILL EN EL ENFOQUE DE COMPETENCIAS
}

\author{
METHOD TO APPLY THE SCHMILL MORAL VALUES TRILOGY \\ ON THE COMPETENCE APPROACH
}

Beatriz E. Terán T.

\section{RESUMEN}

La presente investigación de carácter cualitativo y diseño emergente está enmarcada en el paradigma interpretativo. Su intención se centró en presentar el método para aplicar la Trilogía de Valores Morales de Schmill a través del saber actitudinal de los programas curriculares basados en el enfoque de competencias del Decanato de Ciencias y Tecnología de la Universidad Centroccidental "Lisandro Alvarado", el cual surgió de una investigación más amplia. Se utilizó el método hermenéutico durante todo el proceso investigativo para acercarse al fenómeno de estudio a través de la experiencia docente de la investigadora y los informantes clave conformados por dos (2) profesionales en ejercicio docente de la Universidad Centroccidental "Lisandro Alvarado" (UCLA) y dos (2) de la Universidad Politécnica Territorial "Andrés Eloy Blanco" (UPTAEB). Se interpretó la información recolectada a través de las técnicas de análisis del discurso y la triangulación de fuentes. Asimismo, se asumieron tres categoría en esta investigación: dos (2) categorías emergentes (la aplicación del enfoque de competencias y la inclusión de los valores morales en el currículo universitario) y una (1) categoría preestablecida (la trilogía de valores morales de Schmill). De esta manera el análisis categorial permitió concluir que el método para planificar los procedimientos que conlleven a la adquisición de valores morales, debe cumplir con unas premisas que involucran: a) la transformación universitaria; b) el manejo apropiado del enfoque de competencias; y c) una propuesta didáctica sencilla para transmitir valores morales como la del estudioso Schmill.

PALABRAS CLAVE: Trilogía de valores morales de Schmill, enfoque de competencias, método.

1 Dra. en Ciencias de la Educación, Docente agregado, Decanato de Ciencias y Tecnología de la Universidad Centroccidental "Lisandro Alvarado". beatriz.teran@ucla.edu.ve 


\section{ABSTRACT}

The present research of qualitative character and emergent design is framed in the interpretative paradigm. Its central purpose was to present the method to apply the Trillogy of Moral Values of Schmill through the attitudinal knowledge of the curricular programs based on the competence approach at the Dean of Sciences and Technology of the Centroccidental University "Lisandro Alvarado", which arose from a broader investigation. The hermeneutic method was used throughout the research process in order to get in touch with the phenomenon of study through the teaching experience of the researcher and the informants formed by two (2) teaching professionals from the Centroccidental University "Lisandro Alvarado" and two (2) from the Territorial Politecnic University "Andrés Eloy Blanco". The information collected was interpreted through the techniques of discourse analysis and the triangulation of sources. As well, three (3) categories were worked in this research: two (2) emerging categories (the application of the competence approach and the inclusion of moral values in the university curriculum) and one (1) settled category (Schmill's trilogy of moral values). In this way the categorical analysis allowed to conclude that the design of a method to plan the procedures that lead to the acquisition of moral values, must obey to some premises: a) university transformation; b) the appropriate management of the competence approach; and c) a simple didactic proposal to transmit moral values such as that one of Schmill's.

KEY WORDS: Trilogy of Schmill moral values, competence approach, method.

\section{INTRODUCCIÓN}

Los seres humanos se educan esencialmente para la convivencia en sociedad. De allí que la UNESCO (1996) proponga como base para la educación del futuro: el saber ser, saber hacer y saber convivir. A partir de estos saberes se pretende lograr una educación que capacite, pero también permita la religación amorosa, toda vez que el docente tome conciencia sobre los valores que transmite.

Es necesario entonces que a través de la educación se logren entrelazar los diversos saberes, para lo cual el educador puede valerse del enfoque de competencias como una estructura o principio que permite la organización del currículo (UNESCO, 2006). Al respecto, Salinas E. (2015) señala que la programación curricular funciona, de acuerdo con un proceso pedagógico que permite sistematizar la enseñanza, asumiendo acciones preventivas, así como la organización y administración de recursos y estrategias adecuadas a las diversas situaciones académicas.

En este mismo orden de ideas, la Universidad Centroccidental "Lisandro Alvarado" (UCLA), "decide orientar su currícula hacia la formación integral de un profesional con sensibilidad social y ambiental, basado en el enfoque de com- 
petencias". De esta forma, la programación curricular asumida por la UCLA (2005, p.2) debe guardar estrecha relación con situaciones de aprendizaje integral que involucren el entrelazamiento de los saberes propuestos por la UNESCO.

Al integrar los saberes en la competencia, la definición de ésta integra de manera compleja, dimensiones de tipo axiológico, personal y profesional en las cuales deben formarse los estudiantes. De allí que la competencia sea definida como una práctica compleja y social que expone saberes, destrezas y aptitudes, además de desarrollar en los sujetos especializadas que impliquen cierto grado de tecnicismo o profesionalización (UCLA, 2005). Para efectos de esta aplicación de las competencias, la universidad asume una clasificación que incluye a las competencias genéricas y a las específicas.

$\mathrm{Al}$ respecto, el fenómeno de estudio de esta investigación se ubica concretamente en las competencias asumidas por la UCLA (2005, p.4) como "genéricas interpersonales", las cuales involucran la expresión de emociones y destrezas; además del trabajo en grupo y el "compromiso social o ético" como expresiones de prácticas sociales y el reflejo de valores morales para la vida compartida.

El quehacer docente estrechamente relacionado con la puesta en práctica de actividades que permitan la formación de los estudiantes en el campo educativo, se convierte en la referencia más importante e inmediata para la adquisición no sólo de conocimientos, sino también de valores morales dentro de la universidad. En consecuencia, los programas curriculares, especialmente, aquellos basados en el enfoque de competencias, pueden representar una vía mediante la cual el docente anticipe una proyección de cómo transmitir los conocimientos, pero muy especialmente los valores.

En este sentido, la inclusión de valores morales para la vida compartida, como pieza importante de las competencias genéricas, representa una labor que el docente debe asumir de manera comprometida. Sin embargo, esto puede resultar en ocasiones difícil debido a la gran gama de valores que se manejan; de allí la interrogante: ¿cómo incluir los valores morales en los programas curriculares basados en el enfoque de competencias?

Aunque pueden existir diversas formas para incluir los valores morales en los programas curriculares, en este estudio se toma la idea de Schmill V. (2009), quien plantea la enseñanza de los valores morales haciendo un paralelismo entre éstos con los colores primarios, que combinados trascienden hacia una extensa 
gradación. Así, se reduce la didáctica a tres valores primarios: responsabilidad, respeto y honestidad que al conectarse generan toda una red valorativa compleja trabajada de forma simple.

Los valores morales planteados en forma de trilogía ofrecen al docente una pedagogía sencilla que puede ejercitarse sin dificultades en un currículo basado en el enfoque de competencias. Al respecto, la intención de esta investigación estuvo centrada en presentar el método para aplicar la Trilogía de Valores Morales de Schmill a través del saber actitudinal de los programas curriculares basados en el enfoque de competencias del Decanato de Ciencias y Tecnología de la Universidad Centroccidental "Lisandro Alvarado", el cual surgió de una investigación más amplia.

\section{REFERENCIAS TEÓRICAS}

En la ruta hacia el aprendizaje deseado, los docentes se valen de un plan o programación curricular con el fin de ordenar los aspectos que de una u otra forma, intervendrán durante el proceso de aprendizaje (Salinas, E., 2015). De esta manera, los docentes organizan en un producto final denominado currículo, tanto lo relacionado con los métodos, estrategias, recursos y tiempo que formarán parte del proceso, así como también, el avance de los saberes que el estudiante deberá adquirir.

Aunque pueda haber diversas definiciones de currículo, lo esencial sobre él es su relación inseparable e indiscutible con el proceso educativo y la forma como éste debe desarrollarse. De allí que Coll (citado en Tobón, S. 2008, p.48) define al currículo como "la teoría educativa y la práctica pedagógica, ... lo que se prescribe y lo que realmente sucede en las aulas". En otras palabras, el currículo simplemente es un plan de acciones anticipadas de aquello que se desea lograr durante el proceso de aprendizaje.

La forma de presentar la programación curricular dependerá en gran medida del enfoque que fundamente a la misma. De esta manera, si el enfoque es conductista, la programación será realizada mediante indicadores de logro o desempeño aplicados en la teoría de objetivos conductuales. Por otro lado, si la orientación asumida gira en torno al denominado enfoque de competencias, la programación curricular deberá basarse en la adquisición de saberes entrelazados.

Para lograr las competencias, el docente planifica en el currículo actividades que permitan desarrollar saberes cognitivos, procedimentales y actitudinales. Los 
saberes cognitivo y procedimental, por lo general sólo pueden evidenciarse en casos muy particulares. En este sentido, el estudiante exteriorizará la competencia a través de acciones producto de una necesidad personal o de un requerimiento específico, como por ejemplo, un examen de contenidos, un procedimiento en el que haya que aplicar algún conocimiento adquirido, entre otros.

Con respecto al saber actitudinal, la competencia generada relacionada con la adquisición de valores, puede evidenciarse de forma inmediata y permanente pues las actitudes reflejadas mediante los valores, son inherentes al ser humano y muy difícilmente se pueden ocultar. De allí que, el estudiante puede realizar un procedimiento en un contexto determinado que involucre cómo hacer las cosas, con quién, por qué y cuándo. Todas esas preguntas conducen a una interacción que implica participar en relaciones personales donde inevitablemente se ubican los valores.

La inclusión de las competencias en el currículo universitario lleva a transformar las perspectivas tradicionales en relación con la enseñanza y el aprendizaje, debido a que lo esencial viene dado por la adquisición de procederes para saber y saber hacer y no solo para la acumulación de conocimientos. Esto a su vez conlleva a asumir cambios profundos en la formación universitaria tanto a nivel de planes, recursos, metodologías, evaluaciones, entre otros, los cuales se manejan de forma particular en el enfoque de competencias.

En este mismo orden de ideas, Tobón, S. (2008, p.49) señala que el trabajo del docente no se reduce al solo hecho "enseñar su materia, sino que, junto con todo el resto de los docentes, es corresponsable para que los alumnos alcancen las competencias... y aprendan aquellos contenidos, especialmente los actitudinales y procedimentales, comunes a distintas áreas". Esto de alguna manera, destroza la organización fraccionada del currículo por áreas.

Por otra parte, las competencias no son conceptualizadas o clasificadas por todas las instituciones educativas de la misma manera. Sin embargo, en sentido general, las clasificaciones existentes apuntan hacia la adquisición de unas competencias de tipo genéricas y otras más específicas o profesionales.

En relación con las competencias genéricas, éstas abarcan según Díaz, A. (2006, p.22), las "genéricas para la vida social y personal, y genéricas académicas". Las primeras permiten una práctica ciudadana excelente que abarca aspectos de la vida social e individual como: la ciudadanía, la tolerancia, la comunicación, los valores, entre otros. Las segundas, están relacionadas con aspectos de la 
formación académica tales como: la adquisición de destrezas de lectura y escritura, la adquisición de nociones matemáticas, ciencias, tecnologías y lengua extranjera.

Por su parte, la UCLA (2005) sub-clasifica a las competencias genéricas en tres grupos: a) las instrumentales relacionadas con habilidades cognoscitivas, metodológicas y lingüísticas; b) las interpersonales que posibilitan la participación y comunicación entre los sujetos; c) las sistémicas que suponen una combinación de las dos competencias anteriores con el fin de lograr no sólo el saber cognitivo, sino también la capacidad para sentir emociones. Precisamente el fenómeno de estudio de esta investigación está ubicado en las competencias genéricas interpersonales, pues son éstas las que involucran lo relacionado con la formación de valores en la educación (UCLA, 2005).

Los valores son sumamente importantes para la sociedad de allí que su abordaje desde el punto de vista pedagógico se realiza a través de metas educativas que fundamentan cualquier conocimiento técnico o instrucción (Schmill, V. , 2009). Así, los valores pueden manifestarse únicamente a través de la interacción social y cultural. A razón de esto, es justo considerar a los valores como piezas coadyuvantes en la constitución de todo ente colectivo y de la sociedad en general (Izquierdo C., 2007).

En este punto es importante señalar que no todos los valores son iguales. Al respecto, Dietrich, V. (1983) distingue varios tipos de valores como los intelectuales, los estéticos y los morales; estos últimos son esencialmente personales pues: de un ser impersonal no podremos predecir jamás la bondad o la maldad moral. Ningún cuerpo material, ninguna planta, ningún animal puede ser bueno o malo. Hablando estrictamente, ... sólo las personas reales, sus actos y actitudes son moralmente buenas o malas (p.170).

Asimismo, lo personal de los valores morales implica que éstos estén cargados de responsabilidad humana. No se hace a una persona responsable por carecer de valores como la belleza, la vitalidad o la inteligencia, pero sí por su avaricia, impureza o injusticia (Dietrich, V. 1983). En este sentido, poner en práctica un valor moral como la justicia conlleva a asumir la responsabilidad de ser justo o injusto.

Otro aspecto importante relacionado con los valores morales tiene que ver con la forma como éstos se transmiten de un individuo a otro: por el simple contacto social que se da primeramente en el seno familiar, luego en la escuela y pos- 
teriormente en el campo laboral. De esta manera, las acciones buenas o malas realizada por los miembros de una sociedad son un resumen de valores proactivos y no proactivos que sólo pueden encontrar su equilibrio a través de la educación (Izquierdo, C., 2007).

Definitivamente, la educación formal o informal es un espacio idóneo para trasmitir valores morales a través de vivencias que puedan ser observadas, comunicadas, compartidas, sentidas y discutidas. De allí la importancia que tiene, en primer lugar, que las instituciones educativas asuman el tema de los valores morales en la educación y en segundo lugar, que puedan conocer y aplicar un método sencillo para educar en valores, pues esto último resultaría imposible, de acuerdo con Pérez Esclarín, (citado por Juárez, J., 2002) si los mismos no son integrados al ejercicio de las organizaciones educativas.

En este sentido, Schmill (citado en Terán, B., 2014, p.124) comienza por señalar el desacierto de las instituciones al realizar grandes listas de valores. Esto dispersa al docente, impidiéndole ubicar situaciones que realmente se puedan llevar a la práctica. En consecuencia, el autor plantea reducir la didáctica de los valores morales haciendo una analogía con los colores primarios. Así, del mismo modo que ocurre con los tres colores básicos de cuyas combinaciones emergen toda la gama cromática, también ocurre con los valores.

No se trata de eliminar valores porque sean irrelevantes, sino de reducir su discurso a tres, con el fin de lograr dos propósitos. Uno tiene que ver con hacer de los valores morales, elementos "más asequibles...más cercanos y la posibilidad de transmitirlos con más eficacia”; y el otro está relacionado con la posibilidad de "ver a partir de su aplicación y combinación...todas las conductas deseables y todos los comportamientos que esperamos después de que una persona ha hecho suyos estos valores" (Schmill, citado en Terán, B., 2014, p.124).

De esta manera los valores morales primarios propuestos por Schmill V. (2009) conforman una trilogía constituida por: a) respeto, b) responsabilidad; y c) honestidad. Así, por ejemplo, surge la tolerancia cuando se respetan las diferencias; la puntualidad y el respeto por el tiempo de los demás se convierten en un hábito cuando se practica la responsabilidad; y puedes generar la confianza suficiente para que otros te respeten y crean en ti cuando eres honesto.

La aplicación de la Trilogía de valores morales de Schmill, como se le ha denominado en esta investigación, conforma una propuesta de sólo tres valores morales primarios fáciles de recordar. A partir de ella, se puede generar toda una 
red valorativa que perfectamente puede permitir la adquisición del saber actitudinal planteado en los programas curriculares establecidos bajo el enfoque de competencias. De allí que esta trilogía constituye el fundamento básico teórico de este trabajo investigativo.

\section{ASPECTOS METODOLÓGICOS}

Esta investigación avalada por el Consejo de Desarrollo, Científico Humanístico y Tecnológico de la UCLA, tiene carácter cualitativo y diseño emergente y está enmarcada en el paradigma interpretativo del conocimiento e insertada en el Área Prioritaria de Investigación sobre la Calidad de la Educación, específicamente en lo relacionado con las Estrategias Educativas de las Líneas de Investigación 2016- 2019 propuestas por el Departamento de Estudios Básicos y Sociales del Decanato de Ciencias y Tecnología de la UCLA.

Se utilizó el enfoque interpretativo y el método hermenéutico con el fin de acercarse al fenómeno de estudio desde la teoría y la realidad, por lo que metodológicamente la investigación se asumió bajo una visión paradigmática interpretativa. Al respecto, el paradigma interpretativo o "hermenéutico, fenomenológico", permite una descripción, comprensión y reconstrucción del conocimiento desde las vivencias y sentimientos de "los actores sociales" (UCLA, 2011, p.6).

Adicionalmente, en esta investigación se ha asumido la técnica de análisis del discurso debido a la utilidad de la misma para interpretar la palabra escrita emitida por los seres humanos en la comunicación (González T., et al, 2003). Al respecto, se analizó el discurso de los informantes clave recogido a través de entrevistas escritas que fueron enviadas vía correo electrónico.

Los informantes clave de esta investigación no fueron seleccionados al azar sino más bien por medio de un procedimiento intencional y conveniente (Martínez, M., 2013), tomando en cuenta principalmente: el contexto educativo en que cual laboran (el universitario). Así, los informantes seleccionados fueron cuatro (4) profesionales universitarios en ejercicio docente, dos (2) provenientes de la UCLA y dos (2) de la Universidad Politécnica Territorial Andrés Eloy Blanco" (UPTAEP).

Asimismo, es importante señalar que la metodología de esta investigación estuvo circunscrita a un proceso de triangulación constituido por la confrontación de "diferentes fuentes de datos,... perspectivas teóricas,... [y] observadores" 
que en definitiva permiten el análisis de manera contrastante con el fin de obtener datos más confiables tomando como premisa la diversidad de origen (Martínez, M., 2006a, p.137).

En este sentido, se utilizó la técnica de triangulación de fuentes para llevar a cabo un proceso de categorización que según Galeano M. (2004), implica organizar y agrupar los conceptos o ideas más importantes de los datos de la realidad de los informantes clave, con el fin de formar las categorías del estudio. De esta forma, las categorías quedaron constituidas por una o pocas palabras que permitieron el fácil manejo de la información.

Luego de organizar los datos en categorías, se recurrió nuevamente a la técnica de triangulación para confrontar la teoría y realidad a través de un análisis de los hallazgos. En tal sentido, las unidades de análisis (partes de los discursos seleccionados) fueron analizadas según criterios cualitativos de la investigadora, los cuales incluyeron: tanto las coincidencias discursivas de los informantes, así como los datos no coincidentes aportados de forma única por un solo informante. De esta manera, se logró visualizar claramente el aporte de las entrevistas de manera cruzada.

\section{RESULTADOS}

El resultado final del análisis arrojó tres (3) categorías para este estudio de las cuales dos fueron emergentes (Aplicación del enfoque de competencias y Valores morales en el currículo bajo el enfoque de competencias) y una (1) fue asumida como prestablecida (Trilogía de valores morales de Vidal Schmill conformada por el respeto, la responsabilidad, y la honestidad). En relación con este último aspecto, Martínez M. (2006b, p.133) señala que a pesar de que en una investigación no existen categorías previas, sí se puede partir de "categorías preestablecidas" que el investigador "tiene en su mente" porque "las ha tomado de otras investigaciones".

\section{Categoría aplicación del enfoque de competencias}

En relación con el enfoque de competencias, los informantes clave señalaron la necesidad de aprender sobre este particular debido a "la no disposición y poca o ninguna formación respecto al tema" (informante A). Estos señalamientos apuntan a reconocer el desconocimiento y posible falta de pericia de los docentes para aplicar el enfoque de competencias. 
Resulta interesante mencionar que en las entrevistas no se señaló de forma puntual el tema de los valores al referirse al enfoque de competencias. Sin embargo, los informantes indicaron que el enfoque de competencias "se ocupa... del "saber"... "ser" y... hacer" (Informante C), lo cual permite inferir que las docentes entrevistadas al menos tienen noción sobre la inclusión de valores como uno de los elementos del enfoque de competencias. Esta inferencia se origina debido a la relación conocida entre el "ser" y la adquisición de valores.

Pese a lo anterior, no se observan señalamientos de parte de las docentes entrevistadas en cuanto a una forma específica para integrar los valores en el currículo. Al respecto, CINDA (2009) señala algunas dificultades encontradas en la aplicación del enfoque de competencias: a) existe una renuencia por parte de los docentes con más experiencia para alterar su actividad rutinaria y dejar de considerarse los ejes centrales del proceso educativo a lo cual se le agrega, la necesidad de ejecutar una labor de manera coordinada en equipos de docentes, con el fin de superar obstáculos y contribuir al aprendizaje de manera integrada con otros saberes; $\mathrm{y} b$ ) existen problemas para integrar las competencias genéricas o transversales al currículo y por ende, formar en aspectos actitudinales o valóricos.

Esta investigación permitió conocer que la aplicación del enfoque de competencias exige unos "procesos de transformación curricular" (informante D), los cuales forman parte de una transformación curricular universitaria más amplia, que de acuerdo con Tobón S., (2006), se requiere en el enfoque de competencias, y pasa por ubicarse en un plan de trabajo que involucra: a) docentes de diversas áreas trabajando en conjunto en favor de las necesidades del estudiante; b) integración de saberes; y c) inclusión de aspectos valóricos al currículo.

Obviamente, siendo el enfoque de competencias una manera de desarrollar y organizar el curriculum en base a la integración de saberes, es lógico que se presenten dificultades tanto en su preparación como en su ejecución. Al respecto, atendiendo al adiestramiento que normalmente posee un docente pedagogo en cuanto a la adquisición de habilidades de lectoescritura y realización de planes curriculares, ente otros, es pertinente pensar que los docentes están capacitados para redactar. Se presentan, sin embargo, dos situaciones, primero, no todos los educadores universitarios son pedagogos, y segundo, no todas las personas tienen la misma habilidad para redactar u organizar información hayan o no egresado de un pedagógico. 
Muchos de los profesores universitarios son profesionales de distintas áreas como la ingeniería, arquitectura, medicina, derecho, sociología, entre otras, quienes muy posiblemente adquirieron sus destrezas profesionales bajo enfoques educativos basados en objetivos conductistas y cognitivistas cuya prioridad fue y ha sido la adquisición de conocimientos fragmentados y específicos. De manera que, si se toma en consideración que incluso para un docente pedagogo, la redacción puede resultar dificultosa, debe ser mucho más problemático la adquisición de tal habilidad para un profesional no pedagogo, cuya prioridad lejos de ser la educación integral ha sido la educación especializada.

\section{Categoría Valores Morales en el currículo bajo el Enfoque de Competencias}

Los informantes de esta investigación indicaron que la transmisión de valores ocurre a través de un proceso ejemplificante. En congruencia, Camps (citado por Martínez L. et al, 2008, p.15) señala que "si educar significa dirigir, formar el carácter o la personalidad, llevar al individuo hacia una determinada dirección, la educación nunca puede ser neutral". Por lo tanto, cada quien enseña lo que sabe y lo que es.

Al respecto, Terán B. (2014) señala que la transmisión de valores involucra dos situaciones: a) darse cuenta de que cualquier comportamiento transmite algo al otro; y b) entender que si es necesario enseñar valores de forma explícita porque la unidad curricular lo requiere, es muy importante no sólo conocer el tema de los valores, sino sobre todo, realizar la transmisión de los mismos desde una didáctica sencilla. De allí que Schmill V. (2009) apunte que una idea prudente para enseñar valores puede ser reduciendo su discurso, lo cual puede lograrse a través de un método que lo permita.

$\mathrm{Al}$ respecto, Zarzar C. (2015, p.80) define método como "un camino, un procedimiento, una forma de hacer algo, una serie de pasos que hay que seguir para alcanzar un resultado", los cuales deben ejecutarse de forma lógica y sistemática. En las entrevistas realizadas en esta investigación, una de las preguntas fue: ¿cómo incluye usted los valores morales en el currículo? Obviamente se esperaba una descripción de algún procedimiento utilizado para incluir los valores. Pese a lo anterior, las docentes no hicieron referencia a ningún tipo de metodologías. Al respecto, Nieto y et al, (citado por Rodríguez, T., et al, 1999.) indican que es necesario que los docentes reciban ayuda, adiestramiento y recursos para llevar a la práctica la inclusión de valores en el currículo. 
Pese a lo anterior, cuando se pidió opinión en las entrevistas realizadas, acerca de los beneficios que pudieran lograrse con un plan o método específico para transmitir cada valor moral, sólo una de las informantes consideró esta idea como positiva mientras que las demás se mostraron dudosas al respecto. En este sentido, es necesario mencionar que los docentes se valen de algo llamado planificación para evitar los problemas que puedan causar las improvisaciones durante el desarrollo del ejercicio docente, y en el currículo basado en competencias la planificación no es una excepción.

La planificación siempre ha sido parte importante de todo proceso de aprendizaje formal, incluso el basado en competencias. Desde esta perspectiva, cuando se trata de diseñar actividades que involucren valores, puede ser oportuno el desarrollo de una metodología sencilla que permita planificar, no el valor en sí, sino los procedimientos educativos a través de los cuales se pueda ayudar a los estudiantes a adquirir esos valores.

\section{Categoría Trilogía de Valores Morales de Schmill}

El enfoque de competencias se compone básicamente de la integración de tres saberes: el cognitivo, el procedimental y el actitudinal. Este último, involucra la incursión de valores en las actividades de aprendizaje. Incluir estos valores conlleva a crear instrucciones en las que los estudiantes deben realizar acciones que les permitan obtener un conocimiento, bajo un contexto valorativo de unión, colaboración, comprensión, tolerancia, respeto, compromiso, entre otros.

La pregunta obligada en este punto es icómo incluir en el contexto educativo universitario todos los valores necesarios para la convivencia? Schmill V. (2009) propone la enseñanza de tres valores morales primarios: respeto, responsabilidad y honestidad, que pueden desencadenar otros valores y virtudes mediante una combinación espontánea. La enseñanza de los valores morales primarios representa una trilogía cuya mezcla conlleva a entrecruzar valores que puedan generar toda una red valorativa (ver gráfico 1).

En las entrevistas realizadas se solicitó información específica relacionada con los tres valores que conforman la trilogía de Schmill: respeto, responsabilidad y honestidad. En este sentido, estos valores fueron interpretados a partir del análisis discursivo y la triangulación de fuentes.

El respeto fue interpretado para esta investigación como una condición altruista que implica reconocer y aceptar el valor de la otredad. Tiene mucha 
importancia pues es la base de cualquier relación. La responsabilidad quedó interpretada como una actitud consciente que cada persona manifiesta en el proceder y que permite aceptar las consecuencias del mismo. Su importancia es fundamental pues a través de la responsabilidad se asumen las consecuencias de los propios actos. Finalmente, la interpretación de la honestidad giró en torno a una cualidad que implica pensar y actuar de manera coherente. Este valor se considera de mucha importancia pues le permite al ser humano actuar sin contradicciones en base a la verdad.

Aunque en las entrevistas realizadas los tres valores morales fueron definidos por separado, en la Trilogía de Vidal Schmill se presenta una propuesta de combinación mediante la cual se trata de entrecruzarlos, creando así toda una red valorativa que representa lo fundamental en la trilogía, pues ella es la derivación compleja que nace a partir de la simple combinación de tres elementos.

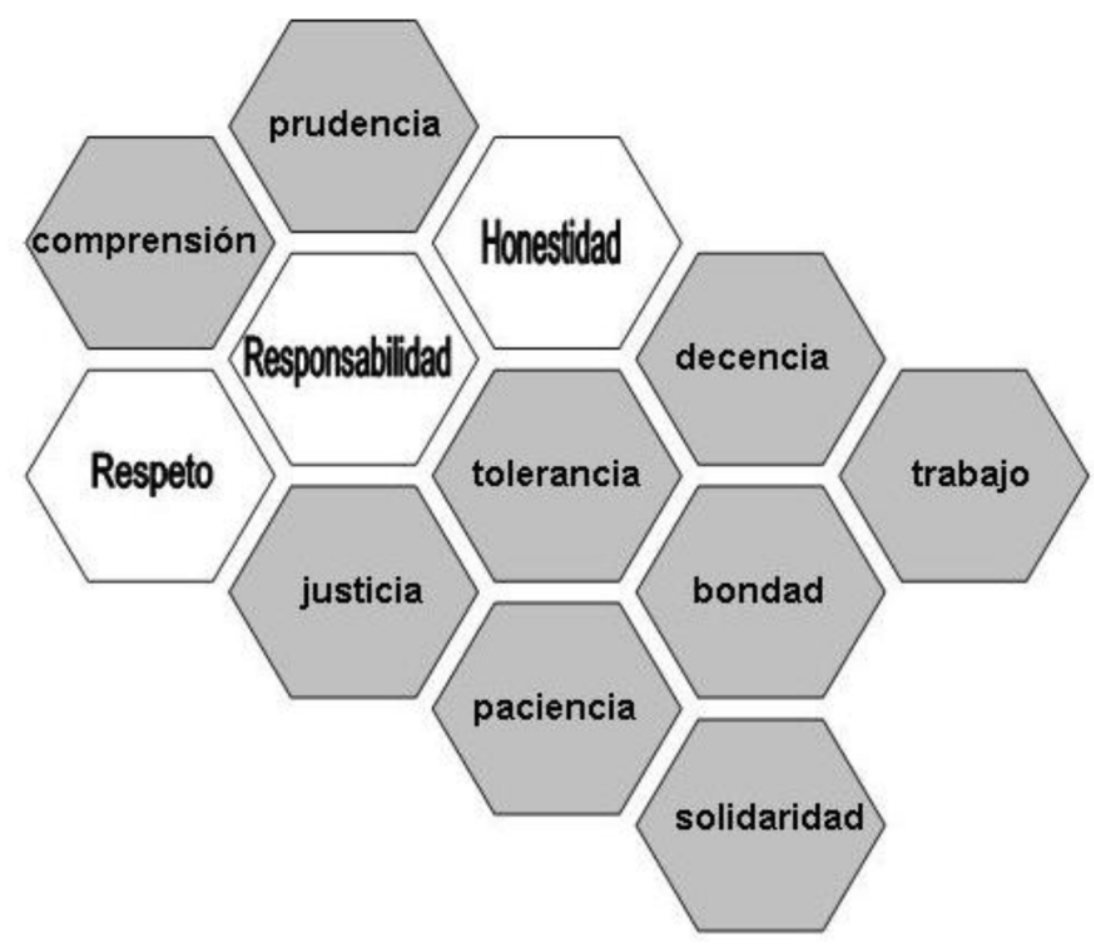

Gráfico 1. Red Valorativa a partir de la Trilogía: respeto, responsabilidad y honestidad (Terán, 2017). 
Precisamente la mezcla de pocos elementos es lo que hace atractiva la propuesta de transmitir los valores morales a partir de la Trilogía de Schmill. En este caso, el docente no tiene por qué ser un experto en valores, ni conocerlos todos de forma erudita para poder incluirlos como parte del currículo universitario, pues a partir de sólo tres, el resto de la combinación se genera de forma casi espontánea.

Asumiendo la red valorativa emergente a partir de la trilogía de Schmill, en el saber actitudinal de los programas curriculares en el enfoque de competencias, se pueden proponer actividades que involucren el aprendizaje del valor moral y a la vez, este mismo aprendizaje pueda generar la adquisición de otros valores. Así, por ejemplo, un saber actitudinal puede quedar redactado de la siguiente forma: "Compartir saberes con los compañeros de clase respetando los diversos puntos de vista sobre las diferencias y similitudes entre el idioma materno y el target language (nuevo idioma a aprender) en relación con la traducción de palabras" (UCLA, 2015).

Del ejemplo anterior, se puede inferir que el estudiante podrá participar en la clase de forma individual o grupal, emitir su punto de vista en relación con el tema y recibir sobre su participación, de manera tolerante, la retroalimentación del docente o de algún compañero. Todo esto implica que el requerimiento realizado en el saber actitudinal, que sólo menciona de forma explícita la acción de compartir y el valor respeto, pueda desencadenar en acciones que involucren otros valores como la comprensión y la paciencia.

En este otro ejemplo, se puede visualizar el mismo fenómeno de red valorativa: "Usar los traductores web con honestidad considerándolos como herramientas para orientar tu propio aprendizaje" (UCLA, 2015). Al interpretar la situación planteada en este saber, se asume que el docente brindará toda su confianza al estudiante para que pueda realizar algunas traducciones utilizando un traductor web, apelando a que su honestidad no le permitirá hacer ningún tipo de trampa, copiando respuestas de otros compañeros mediante el uso del recurso electrónico (móvil, tableta, computadora portátil). En este sentido, no sólo se considera el aprendizaje del valor honestidad, sino también otros involucrados como responsabilidad (consigo mismo) y respeto (hacia la confianza del docente). 


\section{DISCUSIÓN}

Si se asume la trilogía de valores de Schmill como base para la creación del saber actitudinal dentro de los programas curriculares bajo el enfoque de competencias, se contará con un método específico y preciso que marque las pautas en la transmisión de valores morales en la universidad. Sin embargo, antes de llegar al método (ver gráfico 2) es necesario aceptar de forma muy responsable la importancia que tiene la transformación educativa universitaria en el enfoque de competencias. Solo así, se estará en la capacidad de utilizar recursos y establecer metodologías enfocadas en el estudiante como centro de la educación y el docente como guía y servidor.

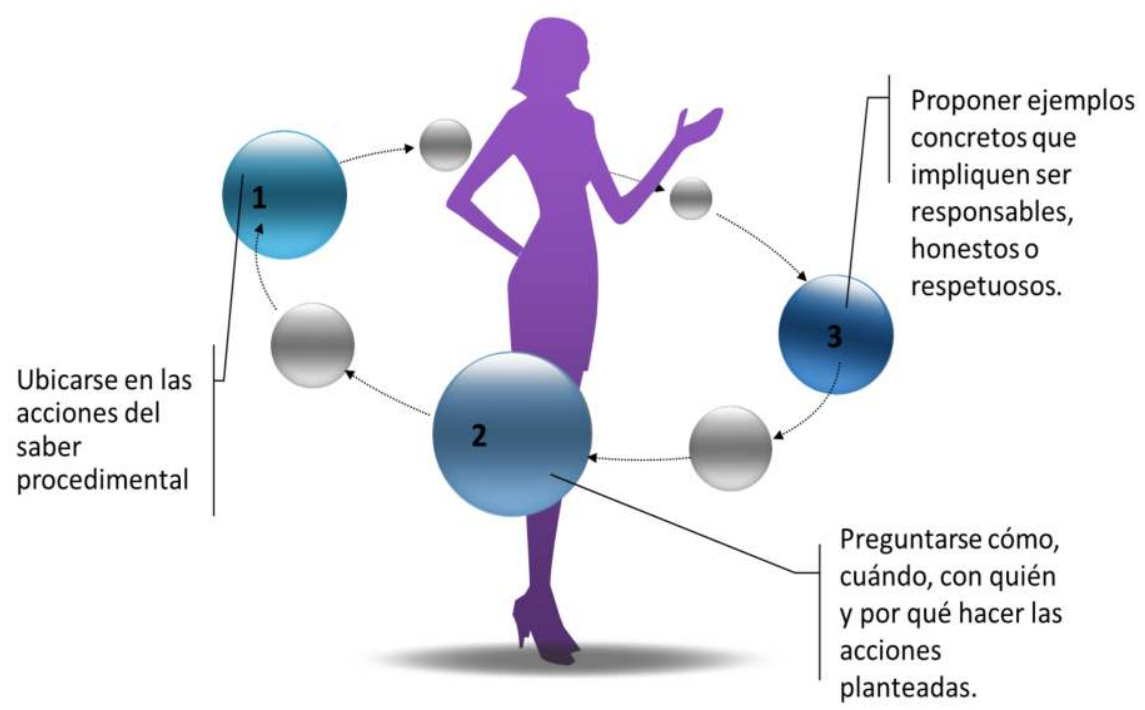

Gráfico 2. Método para aplicar la trilogía de valores morales de Schmill a través del saber actitudinal (Terán, 2017).

Con base a lo anterior, el método propuesto en esta investigación denominado: Método para aplicar la trilogía de valores morales de Schmill a través del saber actitudinal de los programas curriculares basados en el enfoque de competencias, quedó conformado por unas premisas y un procedimiento específico que se describe a continuación.

Las premisas para aplicar el método son las siguientes: a) conocer el enfoque de competencias aplicado a la formación de profesionales y en consecuencia aceptar la 
transformación educativa universitaria generada a partir de la implementación de dicho perfil; b) comprender la diferencia entre los diversos saberes propuestos en el perfil (cognitivo, actitudinal y procedimental), debido a que ubicarse en la actividad procedimental es lo más conveniente a la hora de generar el saber actitudinal. De hecho, puede ser más fácil idear la forma de valorar el procedimiento (el actuar) que el conocimiento (el pensar). El actuar es más evidente, mientras que el pensar es más íntimo; y c) conocer y comprender la Trilogía de Valores Morales propuesta por Vidal Schmill, y la red valorativa que puede generar su combinación.

El procedimiento del método quedó conformado por tres (3) pasos: 1. Ubicarse en las acciones que se desean lograr a partir del saber procedimental. 2. Realizarse preguntas sobre cómo, cuándo, con quién y por qué hacer las acciones planteadas. 3. Proponer ejemplos concretos que requieran de un cierto comportamiento moral en equipo o individualmente y que impliquen ser responsables, honestos o respetuosos del tiempo, los compañeros, los recursos, entre otras situaciones en la universidad.

\section{REFLEXIONES FINALES}

El enfoque de competencias como parte del cambio curricular y nuevo modelo educativo implica: a) reconocer el desconocimiento que se pueda tener sobre tal modelo y la necesidad de recibir adiestramiento para llevarlo a cabo de forma adecuada; b) ubicarse en un plan de trabajo coordinado en equipos de docentes que coadyuven en el desarrollo y organización del currículo en base a la integración de saberes o aspectos intelectuales, procedimentales y actitudinales de manera simultánea y en igual grado de importancia; y c) enfrentar a través de una metodología sencilla, las dificultades que puedan generase a partir de la redacción y organización de aspectos actitudinales valóricos que deben quedar plasmados en el nuevo currículo.

La planificación de los procedimientos o aspectos que conllevan a la adquisición de los valores morales (esenciales en el saber actitudinal), debe responder no sólo a una necesidad y utilidad sino también a unas condiciones mínimas que permitan llevar a cabo el método sugerido, por lo tanto, este método debe cumplir con unas premisas que involucran de manera generalizada: a) asumir la transformación universitaria; b) manejar apropiadamente el enfoque de competencias; y c) disponer de una propuesta didáctica sencilla para transmitir valores morales como la del estudioso Schmill. 


\section{REFERENCIAS BIBLIOGRÁFICAS}

CINDA (2009). Diseño Curricular Basado en Competencias y Aseguramiento de la Calidad en la Educación Superior. Centro Interuniversitario De Desarrollo - Grupo Operativo de Universidades Chilenas Fondo De Desarrollo Institucional. - MINEDUC - CHILE. Disponible:

https://webcache.googleusercontent.com/search?q=cache:Cf_SYpVTWG4J :https://www. cinda.cl/download/libros/39.pdf $+\& c d=1 \& h l=e n \& c t=c \operatorname{lnk} \& g l=v e \& c l i e n t=$ firefox-b.

Díaz, Á. (2006). "El enfoque de competencias en la educación. ¿Una alternativa o un disfraz de cambio?”. Perfiles educativos. vol.28 no.111 México. [7-36]. Disponible:

http://www.scielo.org.mx/scielo.php?pid=s018526982006000100002\&script=sci_arttext.

Dietrich, V. (1983). Ética. Encuentro, Madrid. Disponible:

https://books.google.co.ve/books?id $=$ m9f7ThvF6j4C\&printsec $=$ frontcover $\#_{\mathrm{v}}=$ onepage $\& q \& f=$ false.

Galeano, M. (2004). Diseño de proyectos en la investigación cualitativa. Fondo editorial Universidad. Eafit. Disponible en:

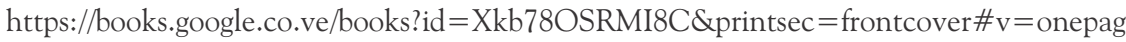
$\mathrm{e} \& \mathrm{q} \& \mathrm{f}=$ false.

González, T. et al (2003). Introducción al análisis de datos en investigación cualitativa: tipos de análisis y proceso de codificación. Tesis de grado Indexada. NURE Investigación no ${ }^{45}$. [110] Disponible en:

http://webcache.googleusercontent.com/search?q=cache:k0k3ym8KYO4J:www.nureinves tigacion.es/OJS/index.php/nure/article/download/485/474 $+\& \mathrm{~cd}=2 \& \mathrm{hl}=\mathrm{en} \& \mathrm{ct}=\mathrm{clnk} \& \mathrm{~g}$ $\mathrm{l}=\mathrm{ve} \& \mathrm{client}=$ firefox $-\mathrm{b}$.

Izquierdo, C. (2007). Valores para vivir en Sociedad. Editorial Paulinas. Caracas Venezuela. Disponible en:

https://books.google.co.ve/books?id=dURdeKOCuvIC\&printsec $=$ frontcover $\#_{\mathrm{v}}=$ onepag $\mathrm{e} \& \mathrm{q} \& \mathrm{f}=$ false.

Juárez, J. (2003). Primeras Jornadas de Educación en Valores: Experiencias religiosas. Universidad Católica Andrés Bello. Caracas. Disponible:

https://books.google.co.ve/books?id =stcY8yFCjg4C\&printsec $=$ frontcover\&dq $=$ Primeras + Jornadas + de + Educaci\%C3\%B3n+en + Valores: + Experiencias + religiosas. \&hl $=\mathrm{en} \& s$

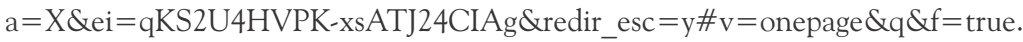

Martínez, L. et al (2008). Educación Física: Transversalidad y Valores. España, S.A. Las Rozas. Madrid. Disponible:

http://books.google.es/books?id $=$ WhOWuW5Vp30C\&printsec $=$ frontcover\&hl $=\mathrm{es}_{\mathrm{v}}=\mathrm{o}$ nepage $\& q \& f=$ false.

Martínez, M. (2006a). "La investigación cualitativa (Síntesis conceptual)”. Revista de investigación en psicología. 135-141 Disponible:

http://sisbib.unmsm.edu.pe/bvrevistas/investigacion_psicologia/v09_n1/pdf/a09v9n1.pdf 
Beatriz E. Terán T.

Martínez, M. (2006b). "La investigación cualitativa (Síntesis conceptual)”. Revista de investigación en psicología. Vol $9 \mathrm{~N}^{\circ} 1$. [123-146] Disponible en:

http://sisbib.unmsm.edu.pe/bvrevistas/investigacion_psicologia/v09_n1/pdf/a09v9n1.pdf.

Martínez, M. (2013). Metodología Cualitativa Integrada 2. Material Audiovisual de Taller sobre Metodología Integrada. 16 al 18 de abril. Universidad Centrooccidental Lisandro Alvarado.

Rodríguez, T. et al. (1999). Valores coincidentes entre los planteamientos educativos de la educación primaria y los de las ONGDs. Ponencia del Cambio educativo, presente y futuro: comunicaciones: VII Congreso Nacional de Teoría de la Educación, Oviedo, 19, 20 y 21 de 1999. Universidad de Oviedo. Rodríguez Teófilo y otros (coordinadores). Disponible: https://books.google.co.ve/books?id=qkccAZxHykMC\&printsec $=$ frontcover\#v $=$ onepag $\mathrm{e} \& \mathrm{q} \& \mathrm{f}=$ false.

Salinas, E. (2015). Tipos de Programación Curricular. Disponible: http://www.academia.edu/6034829/Tipos_de_Programaci\%C3\%B3n_Curricula.

Schmill, V. (2009), julio, 9. ¿Que son los valores? Grabación en Vídeo del taller para los trabajadores de la CFE, México. Disponible:

http://www.youtube.com/watch?v=J1HO387y1vQ\&feature = relmfu.

. (2009), julio, 9. Valores primarios. Grabación en Vídeo del taller para los trabajadores de la CFE, México. https://www.youtube.com/watch?v=bT1NT3oNmUs.

. (2009), octubre, 3. Diferencias y relaciones entre moral y ética. Grabación en Vídeo del taller para los trabajadores de la CFE, México. Disponible en:

http://www.youtube.com $/$ watch? $\mathrm{v}=$ HhJ3S6E7m1k\&feature $=$ related.

Terán, B. (2014) . Ética integral ambiental en la docencia universitaria. Tesis Doctoral. Universidad Fermín Toro. Cabudare. Lara. Venezuela.

Tobón, S. (2006). Formación Basada en Competencias. Pensamiento complejo, diseño curricular y didáctica. ECOE ediciones. Bogotá.

Tobón, S. (2008). La Formación Basada en Competencias en la Educación Superior. El enfoque complejo. Universidad Autónoma de Guadalajara. Curso IGLU. México. Disponible: http://www.youblisher.com/p/192708-revista/.

UCLA. (2005). Directrices para la formulación del perfil profesional bajo el enfoque por competencia. Disponible: http://www.ucla.edu.ve/comision/curriculo/competencia.pdf.

UCLA. (2011). Manual para la elaboración y presentación del Trabajo Especial de Grado, Trabajo de Grado y Tesis Doctoral del Decanato de Ciencias de la Salud de la UCLA. Aprobado en sesión Nro. 2127. Gaceta Universitaria Nro. 126. Disponible:

http://www.ucla.edu.ve/secretaria/Gacetas/GACETAS/GACETA\%20126/Manual\%20par a\%20la\%20Elaboraci\%C3\%B3n\%20y\%20Presentaci\%C3\%B3n\%20del\%20Trabajo\%20Es pecial\%20de\%20Grado.pdf.

UCLA. (2015). Programa de la unidad curricular Inglés II de Ingeniería en Telemática del Decanato de Ciencias y Tecnología. Barquisimeto: Autor. 
UNESCO. (1996). La educación encierra un tesoro. Informe a la UNESCO de la Comisión Internacional sobre la Educación para el Siglo XXI presidida por Jacques Delors. Compendio. Santillana. Ediciones UNESCO. Disponible: http://www.unesco.org/education/pdf/DELORS_S.PDF.

UNESCO. (2006). Revisión de la competencia como organizadora de los programas de formación: hacia un desempeño competente. Observatorio de reformas educativas Universidad de Quebec, Montreal, Canadá. Disponible:

http://www.ibe.unesco.org/es/comunidades/comunidad-de-practica-cop/enfoque-porcompetencias.html.

Zarzar, C. (2015). Métodos y Pensamiento Crítico 1. Grupo Editorial Patria. Disponible en: https://books.google.co.ve/books?id=EtBUCwA AQBAJ\&printsec $=$ frontcover\&source $=$ gbs_ge_summary_r\&cad $=0 \#_{\mathrm{v}}=$ onepage $\& \mathrm{q} \& \mathrm{f}=$ false. 